\section{Large SARS-CoV-2 Outbreak Caused by Asymptomatic Traveler, China}

\author{
Andrei R. Akhmetzhanov \\ Author affiliation: College of Public Health, National Taiwan Uni- \\ versity, Taipei, Taiwan
}

DOI: https://doi.org/10.3201/eid2612.203437

To the Editor: Liu et al. (1) reported on a large outbreak of $>70$ cases of severe acute respiratory syndrome coronavirus 2 (SARS-CoV-2) infection. The origin of the outbreak was traced back to an asymptomatically infected traveler. However, delays in detecting SARS-CoV-2 infections in families B and C1 represent missed opportunities for earlier isolation and interruption of disease transmission.

After reading Lui et al. (1), we questioned whether April 7 was the first day of illness onset for the initial confirmed case, B2.3. Because viral load and infectiousness peak around the time of symptom onset and exposure of family $\mathrm{C} 1$ to case B2.3 was 9 days before that date, presymptomatic transmission would be highly unlikely (2). Although B2.2, who was an asymptomatic carrier, also could have played a role in exposing the family of $\mathrm{C} 1$, a close examination of publicly available records (3) altered this hypothesis.

Exposed on March 26, case B2.3 transmitted the virus to family C1 3 days later, on March 29, which appears to be 1 day before his first symptoms. Case B2.3 went to an outpatient clinic with a subjective fever on March 30 but was not tested for SARSCoV-2. He was not isolated until he went to a clinic again on April 7 with worsening symptoms. Earlier isolation and testing of B2.3 could have prompted earlier contact tracing and triggered earlier diagnosis of C1 during his hospital stay, potentially preventing the chain of $>60$ SARS-CoV-2 transmissions in 2 hospitals.

The uncooperative behavior of cases B2.2 and B2.3 complicated efforts for early contact tracing (3), demonstrating cooperation with medical officers, coupled with proactive case-finding and earlier case isolation, clearly are crucial in curbing disease spread $(4,5)$. If timely actions had been implemented, the outbreak could have been prevented or greatly reduced in size.

\section{References}

1. Liu J, Huang J, Xiang D. Large SARS-CoV-2 outbreak caused by asymptomatic traveler, China. Emerg Infect Dis. 2020;26:2260-3. https:// doi.org/10.3201/eid2609.201798
2. He X, Lau EHY, Wu P, Deng X, Wang J, Hao X, et al. Temporal dynamics in viral shedding and transmissibility of COVID-19. Nat Med. 2020;26:672-5. https:/ / doi. org/10.1038/s41591-020-0869-5

3. Health Commission of Heilongjiang Province China. Patient trajectory: release of new confirmed cases, asymptomatic infection trajectory of 10 April [in Chinese] [cited 2020 Jul 25]. http://yiqing.ljjk.org.cn/index/pcontrol/newsinfo/ id/1823.html

4. Wang CJ, Ng CY, Brook RH. Response to COVID-19 in Taiwan: big data analytics, new technology, and proactive testing. JAMA. 2020;323:1341-2. https://doi.org/10.1001/ jama.2020.3151

5. Dinh L, Dinh P, Nguyen PDM, Nguyen DNH, Hoang T. Vietnam's response to COVID-19: prompt and proactive actions. J Trav Med. 2020;27(3):taaa047. PubMed https:/ / doi. org/10.1093/jtm/taaa047

Address for correspondence: Andrei R. Akhmetzhanov, Global Health Program \& Institute of Epidemiology and Preventive Medicine, College of Public Health, National Taiwan University, No. 17, Xuzhou Rd, Zhongzheng District, Taipei City 100025, Taiwan; email: akhmetzhanov@ntu.edu.tw

\section{Interpreting Transmissibility of COVID-19 in Children}

\author{
Eun Young Cho, Eun Hwa Choi, Jong-Hyun Kim
}

Author affiliations: Chungnam National University Hospital, Daejeon, South Korea (E.Y. Cho); Seoul National University College of Medicine, Seoul (E.H. Choi); College of Medicine, The Catholic University of Korea, Seoul (J-H. Kim)

DOI: https://doi.org/10.3201/eid2612.203452

To the Editor: We read with great interest the article by Park et al. (1) on contact tracing of 5,706 patients with coronavirus disease (COVID-19) during the early phase of the pandemic in South Korea. In the study, the overall detection rate of COVID-19 among household contacts was $11.8 \%$; the highest detection rate $(18.6 \%)$ was in household contacts of those 10 19 years of age and the lowest detection rate (5.3\%) in household contacts of those 0-9 years of age. The media have reported the research as evidence that transmissibility in adolescents and adults is similar (2). Such an interpretation may influence decisionmaking on the reopening of schools. 
Although this study nicely demonstrated the effectiveness of contact tracing strategy during a period of school closure, understanding transmissibility and the implications for the reopening of schools requires reinterpretation of the data. As of April 29, 2020, a total of $37.8 \%$ of the $10-19$ age group were 19 years of age $(223 / 590)$ and, therefore, were not school children (3). A recently published study in South Korea (4) reported 107 primary source children (aged 0-18) had 248 household contacts and only 1 became infected, giving a secondary attack rate of $0.5 \%$. Data from source and contact tracing in the Netherlands (5) also confirmed low transmissibility in children $<18$ years of age $(0 / 43$, $0 \%$ ) compared with persons $\geq 18$ years $(55 / 566,8.3 \%)$.

Accumulating data, including this study, suggest low transmissibility in infected children $<10$ years of age. However, transmissibility in the adolescent age group is unclear at this time. The 10-19 years age group includes diverse students who have completely different contact patterns from elementary school through college; thus, transmission dynamics of COVID-19 may be different. Further detailed studies on understanding transmissibility of the virus by each school level can provide helpful insights for safe reopening of schools.

\section{About the Author}

Dr. Cho is a clinical associate professor at Department of Pediatrics, Chungnam National University Hospital. Her primary research focuses on pediatric infectious diseases.

\section{References}

1. Park YJ, Choe YJ, Park O, Park SY, Kim YM, Kim J, et al.; COVID-19 National Emergency Response Center, Epidemiology and Case Management Team. Contact tracing during coronavirus disease outbreak, South Korea, 2020. Emerg Infect Dis. 2020;26:1666-70. https:/ / doi.org/10.3201/ eid2610.201315

2. Mandavilli A. Older children spread the coronavirus just as much as adults, large study finds. The New York Times. 2020 Jul 18 [cited 2020 Aug 13]. https:/ / www. nytimes.com/2020/07/18/health/coronavirus-childrenschools.html

3. Korea Centers for Disease Control and Prevention. Updates on COVID-19 in Republic of Korea, 29 April 2020 [cited 2020 Nov 13]. https://www.kdca.go.kr/board/ board.es? $\mathrm{mid}=$ a30402000000\&bid=0030\&act=view\&list_ no $=367037$

4. Kim J, Choe YJ, Lee J, Park YJ, Park O, Han MS, et al. Role of children in household transmission of COVID-19. Arch Dis Child. 2020 Aug 7 [Epub ahead of print]. https:/ / doi.org/10.1136/archdischild-2020-319910

5. National Institute for Public Health and the Environment. Children and COVID-19. 2020 Jul 20 [cited 2020 Aug 13]. https://www.rivm.nl/en/novel-coronavirus-covid-19/ children-and-covid-19

Address for correspondence: Jong-Hyun Kim, Department of Pediatrics, St. Vincent's Hospital, College of Medicine, The Catholic University of Korea, 93 Jungbu-daero, Paldal-gu, Suwon 16247, South Korea; email: jh00mn@catholic.ac.kr; Eun Hwa Choi, Department of Pediatrics, Seoul National University Children's Hospital, Seoul National University College of Medicine, 101 Daehak-ro, Jongno-gu, Seoul 03080, South Korea; email: eunchoi@snu.ac.kr 